PLAN DE ACCIÓN PARA PROMOVER PARTICIPACIÓN DE DOCENTES EN REDES Y PUBLICACIONES EN REVISTAS EDUCATIVAS

Esperanza Asencio y Nilda Ibarra (p. 100-123)
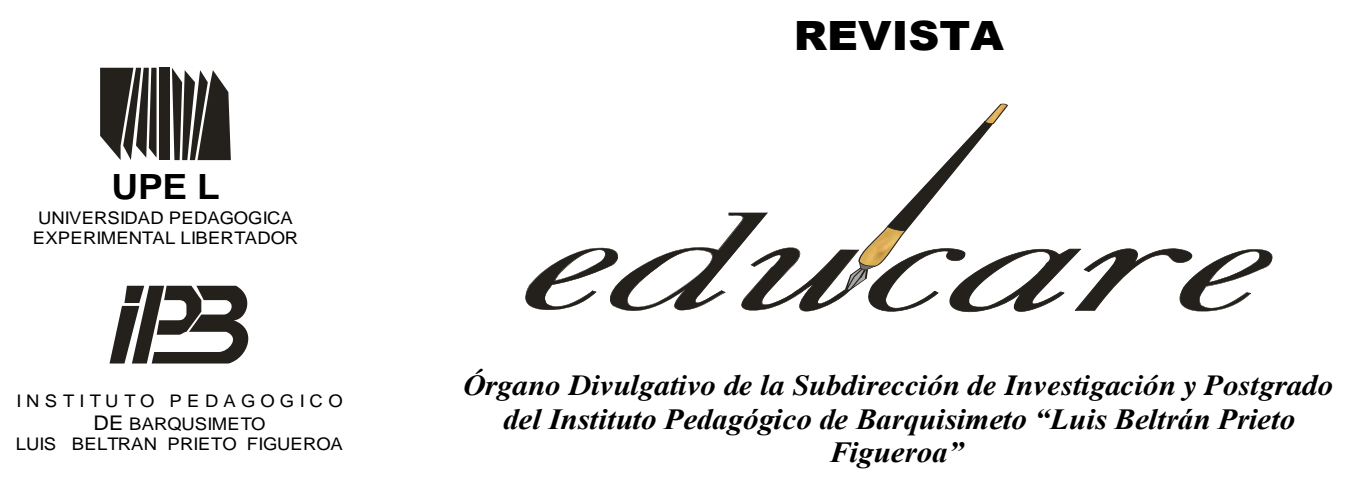

BARQUISIMETO - EDO. LARA - VENEZUELA

NUEVA ETAPA

FORMATO ELECTRÒNICO

DEPOSITO LEGAL:

PPI2O 1002LA3674

VOLUMEN 19 № 3

ISSN: 2244-7296

SEPTIEMBRE - DICIEMBRE 2015

\title{
PLAN DE ACCIÓN PARA PROMOVER PARTICIPACIÓN DE DOCENTES EN REDES Y PUBLICACIONES EN REVISTAS EDUCATIVAS
}

ACTION PLAN TO PROMOTE PARTICIPATION OF TEACHERS IN NETWORKS AND PUBLICATIONS IN EDUCATIONL JOURNALS

\section{ESPERANZA ASENCIO* \\ NILDA IBARRA*}

* UNIVERSIDAD DE CIENCIAS PEDAGÓGICAS “FÉLIX VARELA MORALES" VILLA CLARA CUBA 
PLAN DE ACCIÓN PARA PROMOVER PARTICIPACIÓN DE DOCENTES EN REDES Y PUBLICACIONES EN REVISTAS EDUCATIVAS

\section{ACTION PLAN TO PROMOTE PARTICIPATION OF TEACHERS IN NETWORKS AND PUBLICATIONS IN EDUCATIONL JOURNALS}

\begin{tabular}{|c|c|}
\hline EXPERIENCIA EDUCATIVA & $\begin{array}{l}\text { Esperanza Asencio Cabot" } \\
\text { Nilda Ibarra López } \\
\text { ** } \\
\text { UCP "Félix Varela Morales" }\end{array}$ \\
\hline Recibido:17/08/2015 & Aceptado: 10/11/2015 \\
\hline RESUMEN & ABSTRACT \\
\hline $\begin{array}{l}\text { El trabajo describe la experiencia en la aplicación } \\
\text { de un Proyecto Institucional de Desarrollo } \\
\text { Profesional gestionado en la Sede Pedagógica de } \\
\text { la Universidad Central "Marta Abreu" de Las } \\
\text { Villas, Cuba, dirigido hacia la concepción de un } \\
\text { plan de acción para promover la participación de } \\
\text { los docentes en el trabajo en redes académicas y } \\
\text { científicas y en la publicación de artículos en } \\
\text { revistas educativas reconocidas } \\
\text { internacionalmente. Se destaca la importancia } \\
\text { actual del tema tratado y se precisa el problema a } \\
\text { resolver. Se describe el diseño metodológico } \\
\text { seguido, el cual se basa en el enfoque de la } \\
\text { investigación-acción, como un proceso de } \\
\text { mejoramiento continuo, organizado en una } \\
\text { secuencia de fases que incluyen, las de: } \\
\text { diagnóstico, planificación, implementación y } \\
\text { evaluación. Por último, se analizan los resultados } \\
\text { o hallazgos así como las perspectivas abiertas } \\
\text { para la continuación del trabajo en próximas } \\
\text { etapas. }\end{array}$ & $\begin{array}{l}\text { The paper describes the experience in } \\
\text { implementing an Institutional Professional } \\
\text { Development Project managed at the } \\
\text { Universidad Central "Marta Abreu" in Las } \\
\text { Villas, Cuba, directed toward the design of an } \\
\text { action plan to promote the participation of } \\
\text { teachers at work in academic and scientific } \\
\text { networks and publishing articles in } \\
\text { internationally recognized educational } \\
\text { journals. The current importance of the topic } \\
\text { is highlighted and the problem needs is } \\
\text { defined. The methodological design is } \\
\text { described, which is based on the approach of } \\
\text { action research as a process of continuous } \\
\text { improvement, organized in a sequence of } \\
\text { phases including: diagnosis, planning, } \\
\text { implementation and evaluation. Finally, the } \\
\text { results or findings and open perspectives for } \\
\text { further work in next stages are analyzed. }\end{array}$ \\
\hline $\begin{array}{l}\text { Descriptores: Plan de acción, participación de } \\
\text { docentes, revistas educativas. }\end{array}$ & $\begin{array}{l}\text { Keywords: action plan, participation, } \\
\text { educational journals. }\end{array}$ \\
\hline
\end{tabular}

\footnotetext{
* Doctora en Ciencias Pedagógicas. Profesora Titular Consultante. Asesora científica de la Dirección de Documentación e Información Científico Técnica de la Universidad Central "Marta Abreu" de Las Villas. Cuba. Correo electrónico: easencio@uclv.cu.

** Licenciada en Letras. Máster en Historia y Cultura de Cuba. Profesora Auxiliar. Jefe de Sección de la Dirección de Documentación e Información Científico Técnica de la Universidad Central "Marta Abreu” de Las Villas. Cuba. nibarra@uclv.cu
}

Revista EDUCARE, Volumen 19, Número 3, Septiembre -Diciembre 2015. ISSN: 2244-7296 Página101 


\section{PLAN DE ACCIÓN PARA PROMOVER PARTICIPACIÓN DE DOCENTES EN REDES Y PUBLICACIONES EN REVISTAS EDUCATIVAS \\ Esperanza Asencio y Nilda Ibarra (p. 100-123)}

\section{INTRODUCCIÓN}

El desarrollo acelerado de la ciencia y la técnica en la época actual, en especial los avances de las Tecnologías de la Información y las Comunicaciones (TIC) han producido un fuerte impacto en los procesos de la investigación científica. Las TIC inicialmente se emplearon fundamentalmente en la fase de obtención y verificación de los resultados como instrumento de potenciación de la capacidad, velocidad de cálculo, de diseño, modelación y automatización de sistemas y procesos; hoy día, transforman tanto la fase teórica de generación del conocimiento como la fase de publicación y divulgación de los resultados (Lee \& Torricella, 2009), al propiciar la interacción entre especialistas y el trabajo en equipos, la elevación de la estructuración de la información y la socialización de los resultados individuales.

Justamente, una de las características del desarrollo científico de finales del siglo XX y principios del siglo XXI es “(...) el incremento de diferentes formas de integración horizontal como recurso necesario para generar nuevos conocimientos y tecnologías, las que han permitido la aparición de nuevas formas de generación de los conocimientos científicos a través de las redes de colaboración" (Nuñez, 2005, p.45)

Al respecto, Fainholc (2006) reconoce "que el conocimiento es más valioso si se complementa con el de otros dentro de un entorno que fomente la construcción conjunta" (p.6). De ahí la importancia que en la sociedad actual, tienen las redes globales por su influencia en la dinámica de cooperación entre los centros de investigación en el ámbito mundial, y muy específicamente en las universidades, por su influencia tanto en sus procesos y productos, como en la generación de nuevos conocimientos a partir del desarrollo de actividades de investigación.

Si hay algo que caracteriza a la revolución de las TIC es que construyen un nuevo espacio, definiendo de manera distinta las relaciones de trabajo, las interacciones y los contactos entre las personas y las instituciones (Velásquez, 2007), ya que abren nuevos canales que aceleran los propios procesos de investigación. En general, las redes académicas y científicas conforman equipos de estudio y de trabajo para compartir informaciones, experiencias, documentación y diversos recursos, con el fin de lograr

Revista EDUCARE, Volumen 19, Número 3, Septiembre -Diciembre 2015. ISSN: 2244-7296 Página102 


\section{PLAN DE ACCIÓN PARA PROMOVER PARTICIPACIÓN DE DOCENTES EN REDES Y PUBLICACIONES EN REVISTAS EDUCATIVAS \\ Esperanza Asencio y Nilda Ibarra (p. 100-123)}

objetivos específicos en algún área del conocimiento (Lopera, 2000) y constituyen nuevas formas de organización del trabajo en la ciencia que promueven el incremento de la información científica.

Precisamente, el crecimiento vertiginoso del volumen de la información científica constituye otro rasgo que caracteriza la ciencia en el mundo de hoy; como consecuencia de este aumento se hizo necesario el desarrollo de herramientas computacionales para sistematizar la información y hacerla más accesible, surgiendo de esta forma un conjunto de bases de datos reconocidas internacionalmente por la calidad y visibilidad de las revistas científicas indexadas en ellas.

Relacionado con lo anterior, Miguel (2011) señala que:

Las revistas científicas son uno de los principales canales de comunicación, difusión de los resultados de investigación y de institucionalización social de la ciencia en la mayoría de los campos del conocimiento y su prestigio y grado de influencia en la comunidad científica dependen de su calidad y visibilidad. (p.188)

De lo planteado se infiere la importancia que en los momentos actuales tiene el trabajo en redes y publicaciones en el ámbito de las universidades. Precisamente, este artículo aborda la experiencia en la ejecución de un Proyecto Institucional de Desarrollo Profesional desarrollado en la Sede Pedagógica de la Universidad Central "Marta Abreu" de Las Villas, Cuba, dirigido hacia la concepción e implementación de un plan de acción para promover la participación de los docentes en el trabajo en redes académicas y científicas y en la publicación de artículos en revistas educativas reconocidas a internacionalmente.

\section{DESARROLLO}

Sobre las particularidades del trabajo en redes y publicaciones en las instituciones de la Educación Superior de Cuba

Las universidades que hoy exhiben más prestigio a nivel mundial, son aquellas que además de su función en la formación de profesionales ofrecen altos productos científicos, lo cual finalmente tributa a la excelencia académica de su claustro (Sánchez, 2006), evidenciada por la calidad y visibilidad de sus publicaciones y la participación en redes 


\section{PLAN DE ACCIÓN PARA PROMOVER PARTICIPACIÓN DE DOCENTES EN REDES Y PUBLICACIONES EN REVISTAS EDUCATIVAS \\ Esperanza Asencio y Nilda Ibarra (p. 100-123)}

académicas y científicas, que a su vez constituyen las vías fundamentales para la socialización de los resultados científicos.

Justamente, los procesos de evaluación y de acreditación de las universidades en el mundo contemporáneo, surgen como resultado del interés de las naciones por la elevación de la calidad de los procesos que en dichas instituciones se desarrollan (Suárez, Escalona y Boza, 2012), destacándose en especial lo relacionado con la investigación científica como mecanismo que puede facilitar el análisis de la situación actual y de las líneas futuras en la formación de profesores universitarios (Aramburuzabala, Hernández y Uribe, 2013).

En particular, en Cuba el Ministerio de Educación Superior (MES) estableció el Sistema Universitario de Programas de Acreditación (SUPRA) en las instituciones de la educación superior, como único sistema autorizado para acreditar los procesos, programas e instituciones de la educación superior en Cuba, el cual fue puesto en vigor mediante la Resolución Ministerial No. 150/99 dictada por el Ministro de Educación Superior y Presidente de la Junta de Acreditación Nacional (MES, 2009).

El objetivo general del SUPRA es, en esencia, lograr el mantenimiento y la elevación continua de la calidad de la educación superior cubana a través de la implementación sistemática de la autoevaluación de las instituciones, carreras y programas de maestrías y doctorados, por lo que debe constituir un instrumento de medición cotidiano de la calidad en los procesos referidos.

Entre los patrones de calidad que establece el SUPRA para evaluar los diferentes procesos, se destacan las cuestiones relativas al nivel científico del claustro, entre las que se consideran como aspectos a medir: la participación de los docentes en redes y las publicaciones logradas por estos en revistas pertenecientes a bases de datos reconocidas internacional y nacionalmente. La consideración de estos aspectos se justifica dada su contribución en la elevación de la visibilidad internacional de los resultados obtenidos en los proyectos de investigación, las tesis de maestría y doctorado, las innovaciones y experiencias, entre otros trabajos científicos, así como en la contribución a incrementar la comunicación, el intercambio de información y la colaboración entre las universidades cubanas y las de otros países.

Revista EDUCARE, Volumen 19, Número 3, Septiembre -Diciembre 2015. ISSN: 2244-7296 Página104 


\section{PLAN DE ACCIÓN PARA PROMOVER PARTICIPACIÓN DE DOCENTES EN REDES Y PUBLICACIONES EN REVISTAS EDUCATIVAS \\ Esperanza Asencio y Nilda Ibarra (p. 100-123)}

Con respecto al tema de las publicaciones y con el fin de unificar los criterios de medición, el MES estableció una clasificación para evaluar la calidad de los artículos publicados por los docentes e investigadores universitarios. En este sentido, se instauró la clasificación de las revistas en cuatro grupos que atendiendo al área del conocimiento de la publicación, organizaba las mismas de acuerdo con las bases de datos en que se encontraban indexadas.

Por otra parte, como resultado del perfeccionamiento del proceso de formación doctoral en Cuba, en septiembre de 2011 la Comisión Nacional de Grados Científicos estableció una relación de revistas aceptadas por especialidades como requisito de publicaciones para las defensas de las tesis de Doctor en Ciencias. Posteriormente, con la intención de contar con un instrumento más coherente para la orientación de instituciones, tribunales, tutores y aspirantes para el cumplimiento del requisito de publicaciones, se emite una normativa (MES, 2012) orientada hacia la estandarización de los criterios de aceptación basado en publicaciones, que tuvo como base los grupos de clasificación establecidos por el MES para los procesos de autoevaluación y acreditación.

Como ejemplo de lo anterior, se muestra la tabla 1 donde aparece la organización de los grupos para el caso específico de las Ciencias Pedagógicas, que es el área que nos ocupa en el presente artículo (MES, 2012).

\section{Tabla1}

Grupos de revistas reconocidas por el MES para las Ciencias Pedagógicas

\begin{tabular}{ll}
\hline Grupos & \multicolumn{1}{c}{ Revistas } \\
\hline 1 & Revistas referenciadas en el Web of Science (WoS) \\
& Revistas referenciadas en Scopus \\
2 & Revistas referenciadas en Pascal \\
& Revistas afines disponibles en ScieLO \\
3 & Revistas referenciadas en ISOC y CLASE \\
& Revistas afines disponibles en DOAJ \\
4 & Revistas cubanas de Ciencias Pedagógicas o de otras ciencias que \\
& en su perfil admitan artículos de carácter pedagógico certificadas
\end{tabular}

Revista EDUCARE, Volumen 19, Número 3, Septiembre -Diciembre 2015. ISSN: 2244-7296 Página105 


\section{PLAN DE ACCIÓN PARA PROMOVER PARTICIPACIÓN DE DOCENTES EN REDES Y PUBLICACIONES EN REVISTAS EDUCATIVAS \\ Esperanza Asencio y Nilda Ibarra (p. 100-123)}

\begin{tabular}{ll}
\hline & por el Ministerio de Ciencia y Tecnología \\
5 & Revistas extranjeras de Ciencias Pedagógicas o de otras ciencias \\
que en su perfil admitan artículos de carácter pedagógico \\
acreditadas en sus respectivos países
\end{tabular}

Las cuestiones planteadas evidencian los retos actuales que enfrentan los claustros de las universidades cubanas para mejorar los procesos que se desarrollan en dichas instituciones. Precisamente este artículo se refiere a estos retos para el caso de una universidad pedagógica en el contexto cubano.

Un acercamiento al problema en el contexto de la Sede Pedagógica de la Universidad Central "Marta Abreu" de Las Villas

Las universidades pedagógicas cubanas como parte de las instituciones que conforman el MES, les corresponden asumir los retos a los que se hacía referencia en el apartado anterior. De ahí que los procesos de investigación deben atemperarse a las exigencias actuales, en especial, en lo relacionado con la necesidad de incrementar el intercambio de información y la visibilidad de los resultados científicos mediante la participación de los docentes en redes académicas y científicas y en la publicación de artículos en revistas educativas reconocidas por el MES.

Esencialmente, este artículo aborda esta problemática en el contexto de la Sede Pedagógica de la Universidad Central "Marta Abreu” de Las Villas y analiza el Proyecto Institucional de Desarrollo Profesional dirigido al tratamiento de dicho problema, el cual fue desarrollado por las autoras del presente trabajo, en representación de la Dirección del Centro de Documentación e Información Científico Técnico de la propia universidad.

El proyecto fue ejecutado aproximadamente durante la etapa comprendida entre los años (2012-2014) y estuvo encaminado a la concepción e implementación de un plan de acción para promover la participación de los docentes de la universidad en redes internacionales y publicaciones en revistas educativas reconocidas por el MES.

Para conocer el nivel de partida y precisar la situación problémica con relación a la temática del proyecto se realizó el análisis de documentos relacionados con el trabajo 


\section{PLAN DE ACCIÓN PARA PROMOVER PARTICIPACIÓN DE DOCENTES EN REDES Y PUBLICACIONES EN REVISTAS EDUCATIVAS \\ Esperanza Asencio y Nilda Ibarra (p. 100-123)}

científico de la universidad. Entre los documentos que aportaron mayor información se destacan: el informe de autoevaluación de la universidad presentado en la acreditación nacional realizada en el 2011 y el informe de balance de la ciencia del año 2011.

El informe de autoevaluación referido recogía la información sobre el trabajo desplegado en la institución en los últimos cinco años, es decir desde el 2006-2010 aproximadamente. En este informe no se ofrecen datos acerca de la participación de los docentes en redes, aunque se reconocen las limitaciones en el intercambio científico con otros centros de otras regiones geográficas y se declara el fortalecimiento de este aspecto en el plan de medidas propuesto. Asimismo, en el propio informe se registra el crecimiento en el índice de publicaciones en soporte de Disco Compacto (CD) derivados de memorias de eventos, pero se destaca que el número de publicaciones en revistas nacionales, indexadas y de impacto, aún es muy pobre, así como es muy baja la circulación internacional de las publicaciones de los docentes de la universidad.

Por otra parte, el informe de balance de la ciencia del año 2011, no declara el comportamiento de la participación de los docentes en redes, aunque si precisa la situación de las publicaciones en el año, cuyo comportamiento aparece en la tabla 2, destacándose la no existencia de artículos publicados en revistas de los grupos 1, 2, 3 y 4. Lo anterior puede evidenciarse también en el gráfico 1.

Tabla 2

Publicaciones de los docentes en el año 2011

\begin{tabular}{llllcccc}
\hline Total & En & En & Revistas & G-1 & G-2 & G-3 & G-4 \\
Publicaciones & CD & Revistas & $\begin{array}{l}\text { reconocidas } \\
\text { MES }\end{array}$ & & & & \\
& & & 0 & 0 & 0 & 0 & 0 \\
\hline 42 & 38 & 4 & 0 & & & & \\
\hline
\end{tabular}




\section{PLAN DE ACCIÓN PARA PROMOVER PARTICIPACIÓN DE DOCENTES EN REDES Y PUBLICACIONES EN REVISTAS EDUCATIVAS \\ Esperanza Asencio y Nilda Ibarra (p. 100-123)}

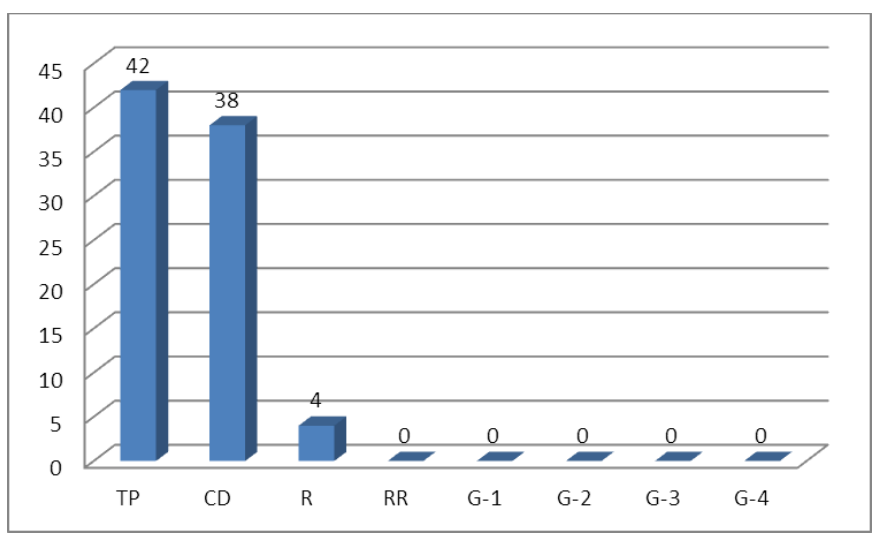

Gráfico 1

Publicaciones de los docentes en el año 2011

En resumen, los documentos analizados evidenciaron las limitaciones que existían en esos momentos en lo que respecta a la participación de los docentes en redes internacionales, así como en publicaciones en revistas reconocidas por el MES.

Por otra parte, se aplicaron diversos métodos empíricos tales como: observaciones, entrevistas a docentes, entrevistas grupales a colectivos departamentales, entre otros, para profundizar en las limitaciones detectadas en el análisis de los documentos. Derivado del estudio realizado se pudo constatar que la mayoría de los docentes de la Sede Pedagógica:

- Desconocían las nuevas formas de construcción y divulgación de los resultados científicos a partir del empleo de las TIC.

- Manifestaban dificultades en el significado de términos tales como: redes académicas y científicas, bases de datos, revistas indexadas, revistas certificadas, revistas arbitradas.

- No estaban actualizados acerca de las exigencias sobre el nivel científico del claustro planteado en las normativas del MES para los procesos de acreditación y formación doctoral.

- Manifestaban poseer un nivel muy pobre en las habilidades tecnológicas que exigía el trabajo en redes virtuales

- Consideraban que sus dificultades eran consecuencias de las limitaciones en el acceso a internet en la institución. 


\section{PLAN DE ACCIÓN PARA PROMOVER PARTICIPACIÓN DE DOCENTES EN REDES Y PUBLICACIONES EN REVISTAS EDUCATIVAS \\ Esperanza Asencio y Nilda Ibarra (p. 100-123)}

- No mostraban interés por publicar en revistas, ya que era más fácil publicar en las memorias de los eventos en soporte de CD.

El análisis de los resultados obtenidos en el estudio realizado permitió definir la situación problémica expresada como: las contradicciones que se manifestaban entre las exigencias planteadas de incrementar el intercambio de información y la visibilidad de los resultados científicos mediante la participación de los docentes en redes académicas y científicas y en la publicación de artículos en revistas educativas reconocidas por el MES y las limitaciones reales que tenían los profesores de la universidad para cumplir esas metas. Derivado de lo anterior fue posible concretar el problema científico alrededor del cual se desarrollarían las tareas del referido proyecto, aspectos que se serán tratados en el apartado que sigue.

\section{Diseño metodológico de la experiencia}

En este epígrafe se analizará el diseño metodológico del Proyecto Institucional de Desarrollo Profesional referido con anterioridad. Es preciso aclarar que el trabajo que se presenta se enmarca en la categoría de experiencias de intervención en la práctica y que aunque se aplican métodos y procedimientos de investigación, no se alcanza el rigor de una investigación científica por lo que no se declara como tal.

Atendiendo a los aspectos analizados previamente se elaboró el diseño metodológico del proyecto, cuyos elementos fundamentales se presentan seguidamente.

Derivada de la situación problémica planteada en el apartado anterior, se declara como problema:

¿Cómo promover el desarrollo profesional de los docentes de la Sede Pedagógica a través del incremento de su participación en redes académicas y científicas y en la publicación de artículos en revistas educativas reconocidas por el MES?

Se consideró como objeto del proyecto el desarrollo profesional de los docentes, mientras que el campo de acción estuvo referido al trabajo en redes internacionales y publicaciones en revistas educativas reconocidas por el MES.

Como objetivo del proyecto fue considerada la elaboración e implementación de un plan de acción que permitiera contribuir a familiarizar a los docentes con las nuevas 


\section{PLAN DE ACCIÓN PARA PROMOVER PARTICIPACIÓN DE DOCENTES EN REDES Y PUBLICACIONES EN REVISTAS EDUCATIVAS \\ Esperanza Asencio y Nilda Ibarra (p. 100-123)}

formas de construcción y divulgación de la ciencia, para promover su participación en redes internacionales y publicaciones en revistas educativas reconocidas por el MES.

Las tareas desarrolladas para dar respuesta al problema fueron las siguientes:

1.- Determinación de los fundamentos teóricos que sustentan el proyecto.

2.- Análisis de la situación actual que presentaban los profesores de la Sede Pedagógica en el tema.

3.- Elaboración del plan de acción.

4.- Validación del plan de acción.

La metodología seguida en el proyecto se enmarca en la perspectiva de la investigación-acción, ya que constituyó un proceso construido desde y para la práctica (Fernández y Calvo, 2012), para incidir en la formación del profesorado posibilitando su mejora continua y la vinculación entre la teoría y la práctica (Romera, 2011). La investigación-acción en general se considera como una espiral de pasos, entre los que se destacan: la planificación, la implementación y la evaluación, que tiene amplias posibilidades como herramienta metodológica para estudiar la realidad educativa, mejorar su comprensión y al mismo tiempo lograr su transformación (Colmenares y Piñero, 2008).

Los actores o sujetos que participaron en la experiencia, según se ha planteado fueron los docentes de la Sede Pedagógica de la universidad de referencia.

\section{Presentación del plan de acción}

Por lo general, el plan de acción se estructura en una serie ordenada de fases, que dependen del objeto a transformar en las que se incluyen las acciones a implementar para lograr el cambio deseado. Es preciso aclarar que en este caso las acciones se fueron construyendo en la práctica, a partir del enfoque metodológico de la investigación-acción, que incluye el apoyo de varias vías procedimentales o técnicas, tanto cuantitativas como cualitativas, para la recogida, sistematización y análisis de las evidencias empíricas; estas fases estuvieron sometidas a ciclos de mejoramiento continuo durante el tiempo de ejecución del proyecto. Seguidamente se describe desde una perspectiva general, la caracterización de las fases consideradas en este caso.

Revista EDUCARE, Volumen 19, Número 3, Septiembre -Diciembre 2015. ISSN: 2244-7296 Página110 


\section{PLAN DE ACCIÓN PARA PROMOVER PARTICIPACIÓN DE DOCENTES EN REDES Y PUBLICACIONES EN REVISTAS EDUCATIVAS \\ Esperanza Asencio y Nilda Ibarra (p. 100-123)}

\section{1.- Fase de diagnóstico.}

En esta fase se retoma el análisis realizado en la determinación de necesidades, profundizándose en aspectos teóricos del tema y en los documentos normativos, así como en cuestiones de orden práctico relacionadas con la búsqueda de información en redes internacionales y en el acceso a bases de datos y revistas; de esta forma, fueron identificadas las redes a nivel internacional que más se ajustaban a los propósitos del plan de acción y se elaboró un repositorio de revistas educativas reconocidas por el MES el cual contenía la información recopilada de cada revista, organizada en carpetas que incluían: la portada, el perfil o presentación, el cuerpo editorial, las normas para colaboradores, las indexaciones, los criterios de evaluación de artículos y los sumarios de los últimos números, entre otros datos.

Por otra parte, se profundizó en el diagnóstico de los docentes al aplicar otros instrumentos, tales como: la observación y las entrevistas en profundidad a docentes, jefes de proyecto y directivos de institución. El análisis de los datos obtenidos permitió concretar la situación real y definir como objetivos del plan de acción los siguientes:

- Contribuir a la familiarización de los docentes con las nuevas formas de construcción y divulgación de la ciencia atendiendo a las exigencias actuales que impone el desarrollo científico-técnico.

- Contribuir al conocimiento de los docentes sobre el trabajo en redes y en la elaboración de artículos.

- Estimular el interés de la universidad por participar en redes internacionales y por publicar en revistas reconocidas por el MES.

\section{2.- Fase de planificación.}

Las acciones del plan fueron estructuradas atendiendo a las vías utilizadas. Así se consideraron las acciones de trabajo metodológico, las acciones de trabajo virtual y las acciones de consultas individuales presenciales. 


\section{PLAN DE ACCIÓN PARA PROMOVER PARTICIPACIÓN DE DOCENTES EN REDES Y PUBLICACIONES EN REVISTAS EDUCATIVAS \\ Esperanza Asencio y Nilda Ibarra (p. 100-123)}

\section{Acciones de trabajo metodológico.}

Se considera oportuno aclarar que en el ámbito de la educación cubana se denomina trabajo metodológico a una instancia de intercambio y de reflexión conjunta que aporta a la formación continuada y al desarrollo profesional de los docentes, el cual se proyecta y ejecuta desde el propio centro educativo, contribuyendo a la preparación de los docentes y su desarrollo profesional. El trabajo metodológico se planifica y organiza en forma sistémica en los diferentes niveles organizativos de la institución escolar atendiendo al diagnóstico de los colectivos de docentes, las necesidades contextuales y las proyecciones de perfeccionamiento de la institución, respondiendo a líneas metodológicas que se van abordando de forma sistémica durante una etapa determinada.

Justamente las potencialidades mencionadas, permitieron que desde el trabajo metodológico de los departamentos docentes se insertaran actividades de capacitación dirigidas esencialmente a contribuir a la actualización de conocimientos y la orientación afectiva hacia los temas siguientes:

- El impacto de las TIC en la investigación científica.

- Las redes académicas y científicas. Ejemplos de redes internacionales en el área educativa.

- Bases de datos y revistas científicas. Ejemplos de bases de datos y revistas educativas reconocidas por el MES.

\section{Acciones de trabajo virtual.}

La creación de una red virtual de apoyo al trabajo del plan de acción contribuyó a crear una nueva dinámica que posibilitó la comunicación simultánea y el intercambio de información y reflexiones con todos los docentes de la sede. Desde esta red:

- Se divulgaron entre los docentes las convocatorias de solicitud de colaboraciones en revistas indexadas, eventos, seminarios, cursos virtuales y otras cuestiones de interés.

- Se crearon listas de discusión y foros en diversos temas que permitieron consolidar las actividades de trabajo metodológico realizadas. 


\section{PLAN DE ACCIÓN PARA PROMOVER PARTICIPACIÓN DE DOCENTES EN REDES Y PUBLICACIONES EN REVISTAS EDUCATIVAS \\ Esperanza Asencio y Nilda Ibarra (p. 100-123)}

- Se realizaron actividades interactivas de consulta, asesoramiento y revisión de manuscritos de los docentes para su envío a revistas.

- Se aplicaron instrumentos para la obtención de datos y para el desarrollo del proceso reflexivo.

- Se divulgaron entre todos los docentes, mensajes de felicitación para estimular los logros individuales obtenidos, fundamentalmente en la publicación de artículos en revistas educativas reconocidas por el MES.

Acciones de consultas individuales de forma presencial.

Entre las acciones del plan de acción se incluyeron consultas presenciales a profesores las cuales estuvieron dirigidas fundamentalmente al desarrollo de habilidades prácticas para acceder a la web y trabajar en redes virtuales. Asimismo desde las consultas, se apoyó la búsqueda e identificación de revistas educativas reconocidas por el MES cuyo perfil se correspondían con las líneas de investigación de los docentes, así como se trabajó en la asesoría y revisión de manuscritos con vista al envío de colaboraciones para posibles publicaciones.

\section{3.- Fase de implementación.}

La implementación del plan de acción transcurrió de acuerdo a lo planteado en la fase anterior. Las acciones de trabajo metodológico en los departamentos se desarrollaron con calidad con una buena asistencia y participación de los docentes. Además, se impartieron otras actividades que no estaban proyectadas del plan de acción, entre las que se destacan: reuniones metodológicas a nivel de universidad, talleres a directivos y miembros de órganos asesores, conferencias en la celebración del "Día de la Ciencia" (2013), en los eventos de Ciencias de la Educación y Pedagogía Provincial (2012) y en el curso Postdoctoral (2013), el curso de líderes científicos (2014) y el curso de Educación Especial (2014).

Asimismo, las acciones virtuales se cumplieron de forma satisfactoria. Se destaca el impacto favorable que tuvo entre los docentes la divulgación de convocatorias de solicitud 


\section{PLAN DE ACCIÓN PARA PROMOVER PARTICIPACIÓN DE DOCENTES EN REDES Y PUBLICACIONES EN REVISTAS EDUCATIVAS \\ Esperanza Asencio y Nilda Ibarra (p. 100-123)}

de colaboraciones en revistas reconocidas por el MES. En la tabla 3 se presenta un resumen de las convocatorias divulgadas por esta vía y los temas tratados.

Tabla 3

Convocatorias divulgadas a través de la red de docentes

1. Revista RIE 61 ( Cooperación internacional, 2012)

2. Revista REICE (Efectos escolares, 2012).

3. Revista RIE 62 (El cuerpo en la escuela, 2013).

4. Cuadernos de investigación educativa (Temas pedagógicos, 2013).

5. Revista Perspectiva Educacional (Las tecnologías en la educación, 2013).

6. Revista RIEE (Evaluación educativa, 2013).

7. Revista Profesorado (Currículo y formación docente, 2013).

8. Revista RIE 63 (Calidad de vida de las personas con discapacidad, 2013).

9. Revista Universidad y Sociedad (Temas pedagógicos, 2013).

10. Revista Páginas de Educación (Temas pedagógicos, 2013).

11. Revista Avances en Supervisión educativa 19 (Temas pedagógicos, 2013).

12. Revista RIEE (evaluación y convivencia entre escolares, 2013).

13. Revista Tecnología (Temas pedagógicos, 2013).

14. Revista RIJS (Educación y justicia social, 2014).

15. Revista perspectiva educacional (Formación de docentes, 2014).

16. Revista RIE 64 (Evaluación educativa, 2014).

17. Revista EDUTEC (Nuevos escenarios de aprendizaje, 2014). 


\section{PLAN DE ACCIÓN PARA PROMOVER PARTICIPACIÓN DE DOCENTES EN REDES Y PUBLICACIONES EN REVISTAS EDUCATIVAS \\ Esperanza Asencio y Nilda Ibarra (p. 100-123)}

18. Revista RIE 65 (Las tecnologías en educación, 2014).

19. Revista REICE (Práctica del liderazgo, 2014).

20. Revista RIEE (Educación Especial y Pre-escolar, 2014).

21. Revista Avances en Supervisión educativa 20 (Temas pedagógicos, 2014).

22. Revista RIJS (Liderazgo educativo y justicia social, 2014).

23. Revista RIE 66 (Entornos escolares saludables, 2014).

24. Revista Profesorado (próximos temas, 2014).

25. Revista de Educación Inclusiva (investigaciones actuales, 2014).

26. Revista de estudios de desarrollo (Desarrollo social y local, 2014).

27. Revista Avances en Supervisión educativa (Recursos educativos, 2014).

28. Revista RIE 67 (los modelos de gestión en centros educativos, 2014).

Por otra parte, las consultas individuales se desarrollaron según lo proyectado. En especial, se reconoce por los docentes el apoyo brindado en la búsqueda de información de revistas, así como en la asesoría y revisión de manuscritos. Se considera importante destacar la ayuda que el repositorio brindó en la atención individual a los profesores, pues de forma rápida y sin necesidad de conectarse a internet fue posible seleccionar y obtener directamente la información de las revistas que se correspondían con sus líneas de investigación, con vista a elaborar artículos para su posible publicación. Además, derivado del trabajo de consulta con los profesores se emprendió la búsqueda de otras revistas, que a su vez enriquecieron de manera sistemática el propio repositorio, el cual en los momentos en que se redactaba este trabajo contaba con más de 100 revistas identificadas por bases de datos.

El trabajo realizado visto desde una perspectiva de desarrollo para próximas etapas, se considera pueda servir de base para la creación de un sitio web (en el que de hecho ya se 


\section{PLAN DE ACCIÓN PARA PROMOVER PARTICIPACIÓN DE DOCENTES EN REDES Y PUBLICACIONES EN REVISTAS EDUCATIVAS \\ Esperanza Asencio y Nilda Ibarra (p. 100-123)}

están desplegando algunas acciones en su programación), que ofrezca a los docentes la información recopilada en el repositorio, de manera que se posibilite la navegación desde cada una de las bases de datos a revistas educativas indexadas en ellas, para obtener información desde la propia plataforma del sitio, sin necesidad de que el usuario esté conectado a Internet, así como que brinde los enlaces correspondientes para la profundización y actualización de la información.

\section{4.- Fase de evaluación.}

Esta fase fue concebida con el propósito de obtener la retroalimentación necesaria del proceso con vista a su mejoramiento continuo, por lo cual fue desarrollada en todas las fases mencionadas con anterioridad. Entre los elementos considerados se debe destacar la valoración satisfactoria que se obtuvo de la aplicación del criterio de expertos a través de rondas en diferentes momentos del plan de acción.

Además, el proceso estuvo sometido a un control sistemático mediante la observación, entrevistas, análisis de documentos, registros de autoevaluación, entre otros instrumentos aplicados a través de diversas vías, que permitieron recoger valoraciones acerca de las acciones que se estaban desarrollando.

\section{Discusión de los principales hallazgos}

Los resultados obtenidos durante la implementación de la estrategia, fueron determinados a partir del análisis de los datos recopilados durante la experiencia. Como se ha planteado la evaluación de las acciones desarrolladas se fueron controlando durante todo el proceso vivido, lo que permitió recoger datos cualitativos a partir de los instrumentos aplicados, los cuales estuvieron dirigidos a comprobar el cumplimiento de los objetivos

trazados en el plan de acción. Para ejemplificar lo anterior, seguidamente se muestran algunos criterios planteados por los docentes:

"Considero como muy interesante lo aprendido sobre las revistas científicas de la web, que me será para poder realizar publicaciones”.

"Pude obtener conocimientos de cómo socializar nuestros resultados de investigación y hacerlos llegar a todos". 


\section{PLAN DE ACCIÓN PARA PROMOVER PARTICIPACIÓN DE DOCENTES EN REDES Y PUBLICACIONES EN REVISTAS EDUCATIVAS \\ Esperanza Asencio y Nilda Ibarra (p. 100-123)}

"Aprendí contenidos novedosos que nos sirven para profundizar y esclarecer dudas que nos permiten introducirnos en el mundo científico”.

"Nos puso en contacto con vías, fuentes y contenidos muy actuales, por lo que pueden ser muy útiles para enriquecer nuestra investigación”.

"Nos sentimos motivados para incorporarnos a redes y para intentar elaborar artículos para revistas importantes”.

"Considero que ayuda mucho la divulgación de convocatorias de revistas que se hace a través de la red de docentes”.

"Nos pone al tanto de lo que está sucediendo en el mundo con respecto a la investigación y el uso de las nuevas tecnologías”.

"Lo que me pareció más interesante fue el acceso a las revistas y a los sitios, por que no sabíamos como llegas hasta ahi y algunos no sabíamos que existían”.

“Aprendimos a buscar información más actualizada, a acceder a revistas de impacto y nos motivaron para participar en redes y publicar artículos científicos”.

En resumen, los datos analizados permitieron evidenciar que los docentes controlados durante la experiencia:

- Se familiarizaron con las nuevas formas de construcción y divulgación de la ciencia atendiendo a las exigencias actuales que impone el desarrollo científico-técnico.

- Conocieron los conceptos fundamentales relacionados con las redes y las publicaciones en revistas reconocidas por el MES.

- Se interesaron por participar en redes internacionales y por publicar en revistas reconocidas por el MES.

Por otra parte, fueron obtenidos otros datos cualitativos y cuantitativos a partir del análisis de los informes anuales de balance de la ciencia en el 2012, 2013 y 2014 que serán discutidos a continuación.

Con relación al trabajo en redes internacionales, en los informes se apreció un incremento en la incorporación de docentes a la Red de la Organización de Estados Iberoamericanos (OEI), los cuales participaron en varios grupos de trabajo (Enseñanza de las Ciencias, Ciencia-Tecnología-Sociedad, Educación para la Sostenibilidad, Educación 


\section{PLAN DE ACCIÓN PARA PROMOVER PARTICIPACIÓN DE DOCENTES EN REDES Y PUBLICACIONES EN REVISTAS EDUCATIVAS \\ Esperanza Asencio y Nilda Ibarra (p. 100-123)}

Infantil y otros) y algunos de ellos elaboraron su blog en la plataforma de la red donde mostraron los resultados de su actividad científica; además los docentes incorporados accedieron a salas de lectura, boletines, foros y otros recursos que esta red le brinda a sus miembros.

Asimismo, se observó que varios profesores se incorporaron a la Red Iberoamericana de Comunicación y Divulgación de la Información Científica y a la Red Iberoamericana de Investigación sobre Cambio y Eficacia Escolar (RINACE), los que participaron en las actividades correspondientes, favoreciendo de esta forma la interacción con docentes de otras partes del mundo, así como obtener informaciones actualizadas del ámbito educativo internacional.

Con respecto a las publicaciones se advierten discretos avances en la producción de artículos científicos por parte de los docentes; lo anterior se puede constatar a partir de los datos obtenidos en los informes de balance, los cuales se muestran en la tabla 5, donde se evidencia que existe una tendencia creciente en el número de artículos publicados en revistas de los grupos 1, 2, 3 y 4 (G-1, G-2, G-3, G-4). Lo anterior también se demuestra en el gráfico 2, en el cual las barras azules representan el año 2012, las rojas el 2013 y las de color verde las del 2014; de forma análoga el gráfico 3, que expresa la evolución de la cantidad de artículos publicados en Revistas Reconocidas por el MES (2011 - 2014).

Tabla 5

Publicaciones de los docentes en 2012, 2013 y 2014

\begin{tabular}{lllllllll}
\hline Año & Total & En & En & Revistas & G-1 & G-2 & G-3 & G-4 \\
& Publicaciones & CD & Revistas & $\begin{array}{l}\text { reconocidas } \\
\text { MES }\end{array}$ & & & & \\
& & & & & & & & \\
\hline 2012 & 87 & 60 & 20 & 7 & 0 & 0 & 1 & 6 \\
2013 & 126 & 84 & 20 & 22 & 1 & 0 & 9 & 12 \\
2014 & 148 & 90 & 30 & 28 & 0 & 2 & 12 & 14 \\
\hline
\end{tabular}

Revista EDUCARE, Volumen 19, Número 3, Septiembre -Diciembre 2015. ISSN: 2244-7296 Página118 


\section{PLAN DE ACCIÓN PARA PROMOVER PARTICIPACIÓN DE DOCENTES EN REDES Y PUBLICACIONES EN REVISTAS EDUCATIVAS \\ Esperanza Asencio y Nilda Ibarra (p. 100-123)}

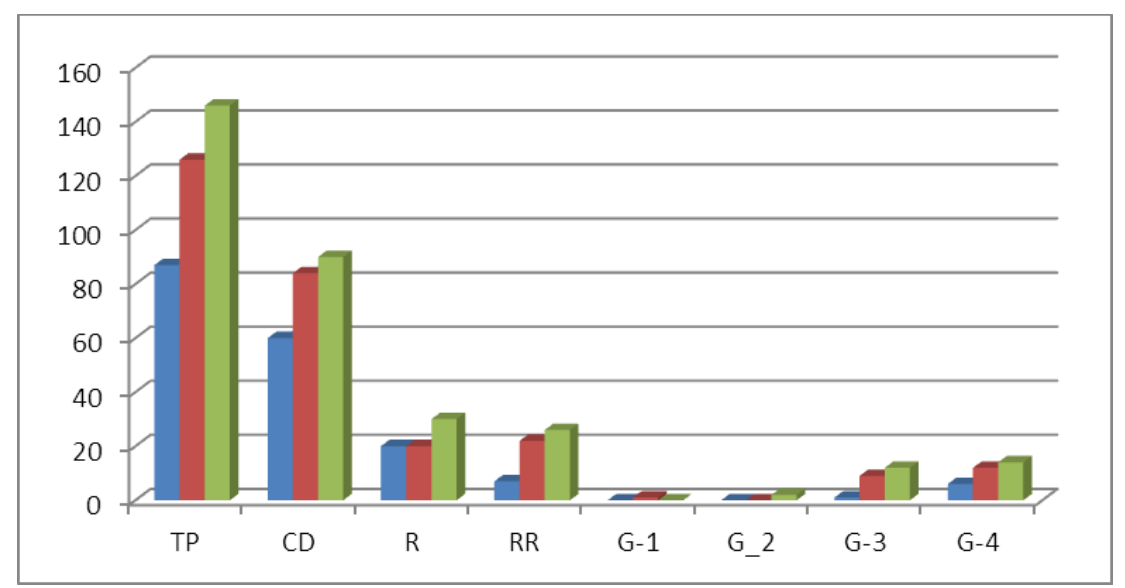

Gráfico 2

Publicaciones de los docentes en 2012, 2013 y 2014

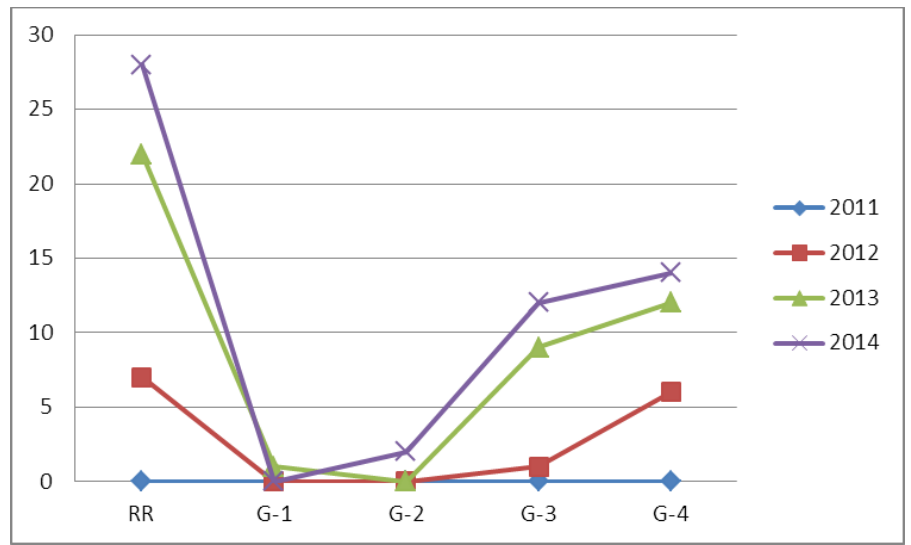

Gráfico 3

Evolución de la cantidad de artículos publicados en revistas Reconocidas por el MES (2011 - 2014)

Además, entre los logros es atinado mencionar que seis revistas indexadas en bases internacionales tienen entre sus expertos o revisores a docentes de la universidad. Se considera oportuno destacar también que el trabajo del proyecto fue reconocido en el plan de estímulos de la universidad otorgado por el rector en el año 2014, al obtener el Premio Anual al Mérito Científico en la categoría de resultado que refleja el avance científico de mayor trascendencia y originalidad.

Resumiendo, el análisis realizado permitió constatar los resultados favorables alcanzados en el cumplimiento de los objetivos del plan de acción aplicado en el marco del Proyecto Institucional de Desarrollo Profesional. Sin embargo, se reconoce que lo logrado 


\section{PLAN DE ACCIÓN PARA PROMOVER PARTICIPACIÓN DE DOCENTES EN REDES Y PUBLICACIONES EN REVISTAS EDUCATIVAS \\ Esperanza Asencio y Nilda Ibarra (p. 100-123)}

es solo un acercamiento a las metas propuestas, ya que existen muchas dificultades en el claustro, fundamente en lo relacionado con la elaboración de artículos, lo cual fue constatado durante la aplicación de la experiencia.

En este sentido, el trabajo desplegado permitió demostrar los problemas que tienen los docentes desde la propia concepción de la estructura del artículo, la cual por lo general responde a más a una ponencia que a un artículo de corte científico; esto es consecuencia de una cultura instalada durante muchos años que privilegió la publicación de ponencias en $\mathrm{CD}$, lo cual fue señalado en este trabajo. Por otro lado, fueron evidenciadas dificultades en lo referido a la actualización y diversificación de las fuentes de información utilizadas y en su asentamiento, así como en la redacción y uso de las reglas ortográficas, entre otros aspectos deficientes.

En general, la identificación de estas necesidades sirvió como antecedente para la propuesta de un sistema de superación profesional dirigido a la preparación de los docentes universitarios para lograr la publicación de artículos científicos en revistas educativas reconocidas internacionalmente, el cual está actualmente en proceso de desarrollo.

\section{CONCLUSIONES}

En este trabajo se presentó la experiencia en la ejecución de un Proyecto Institucional de Desarrollo Profesional dirigido hacia la concepción y aplicación de un plan de acción para promover el intercambio de información y la visibilidad de los resultados científicos mediante la participación de los docentes en redes académicas y científicas y en la publicación de artículos en revistas educativas reconocidas por el MES.

El plan de acción constituyó un proceso construido desde y para la práctica, que utilizó como herramienta metodológica el enfoque de la investigación-acción, para incidir en la actualización de la preparación del profesorado. Dicho plan fue estructurado en una serie ordenada de fases (diagnóstico, planificación, implementación y evaluación) sometidas a ciclos de mejoramiento continuo, que incluyó el apoyo de varias vías procedimentales y técnicas.

Revista EDUCARE, Volumen 19, Número 3, Septiembre -Diciembre 2015. ISSN: 2244-7296 Página120 


\section{PLAN DE ACCIÓN PARA PROMOVER PARTICIPACIÓN DE DOCENTES EN REDES Y PUBLICACIONES EN REVISTAS EDUCATIVAS \\ Esperanza Asencio y Nilda Ibarra (p. 100-123)}

El diagnóstico realizado permitió definir el nivel de alcance del plan de acción atendiendo a la situación real de los docentes de la Sede Pedagógica, por lo que fueron consideradas acciones de familiarización con las nuevas formas de construcción y divulgación de la ciencia que contribuyeran a su conocimiento y a estimular su interés por participar en redes y publicar artículos en revistas educativas.

Las acciones contempladas en el plan fueron concretadas a partir del trabajo metodológico, el trabajo virtual y las consultas individuales presenciales. Estas acciones en general, contribuyeron al logro de un despegue en la participación de docentes a redes y en su toma de conciencia acerca de la necesidad de elevar el rigor y la calidad en las producciones derivadas de los resultados de las investigaciones para alcanzar la publicación de sus trabajos en revistas reconocidas por la comunidad científica.

En la próxima etapa, se pretende continuar trabajando en esta línea mediante un sistema de superación profesional intencionado a la elevación de la preparación de los docentes en lo referido a la gestión de la información científica en las condiciones actuales. Asimismo se proyecta perfeccionar el trabajo con la red virtual a partir de la creación de una plataforma interactiva, que contribuya al trabajo colaborativo en línea y al intercambio de experiencias entre los docentes en los temas referidos a la actualización de la información científico técnica. De igual forma, se piensa incorporar estas ideas en la formación inicial de docentes para dar respuesta a los desafíos que imponen la informatización de la sociedad cubana.

\section{REFERENCIAS}

Aramburuzabala, P., Reyes Hernández-Castilla, R. y Ángel-Uribe I.C. (2013). Modelos y tendencias de la formación docente universitaria. Revista Profesorado, 17 (3). Fecha de consulta: 2014-09-20. Disponible: http://www.ugr.es/local/recfpro/rev173COL9.pdf

Colmenares, M. y Piñero M. L. (2008). La investigación acción: una herramienta metodológica heurística para la comprensión y transformación de realidades y prácticas socio-educativas. Revista Laurus, 14 (27), 96-114. Fecha de consulta: 2014-09-20

Revista EDUCARE, Volumen 19, Número 3, Septiembre -Diciembre 2015. ISSN: 2244-7296 Página121 


\section{PLAN DE ACCIÓN PARA PROMOVER PARTICIPACIÓN DE DOCENTES EN REDES Y PUBLICACIONES EN REVISTAS EDUCATIVAS \\ Esperanza Asencio y Nilda Ibarra (p. 100-123)}

Disponible: http://redalyc.uaemex.mx/src/inicio/ArtPdfRed.jsp?iCve=76111892006

Fainholc, B. (2006). Rasgos de las universidades y de las organizaciones de educación superior para una sociedad del conocimiento, según la gestión del conocimiento. Revista de Universidad y Sociedad del Conocimiento (RUSC), 3(1). Fecha de consulta: 2013-10-7. Disponible: http://journals.uoc.edu/ojs/index.php/rusc/article/view/v3n1-fainholc

Fernández, E. y Adelina Calvo, A. (2012). La formación permanente del profesorado en el uso innovador de las TIC. Revista Profesorado, 16 (2).

Fecha de consulta: 2014-09-20. Disponible: http://www.ugr.es/local/recfpro/rev162COL7.pdf

Lee, F. y Torricella, R. (2009). Estrategias Web de Publicaciones Científicas. Conferencia en reunión nacional con editores de revistas de universidades pedagógicas. La Habana. Cuba: MES.

Lopera, H. (2000). Integración de redes de conocimiento: una responsabilidad de la biblioteca universitaria. Ponencia presentada en el Sexto Congreso Nacional de Bibliotecología y Documentación, ASCOLBI. Santafé de Bogotá, 2000. Fecha de consulta: 2012-04-15. Disponible: http://caribe.udea.edu.co/ hlopera/irc.html

MES (2009). Resolución Ministerial No. 150/99. Dictada por el Ministro de Educación Superior y Presidente de la Junta de Acreditación Nacional. La Habana: Editado por el Ministerio de Educación Superior cubano.

MES (2012). Normativa para la aprobación de publicaciones científicas. La Habana: Editado por la Comisión Nacional de Grados Científicos.

Miguel, S. (2011). Revistas y producción científica de América Latina y el Caribe: su visibilidad en SciELO, RedALyC y SCOPUS. Revista Interamericana de Bibliotecología, vol. 34, no. 2, p. 187-199.

Núñez, J. (2005). La Ciencia y la Tecnología como procesos sociales. La Habana: MINED. $98 \mathrm{p}$.

Romera, M.J. (2011). La investigación-acción en la formación del profesorado. Revista Española de Documentación Científica, 34 (4), 597-614. DOI: 10.3989/redc.2011.4.836

Sánchez, B. (2006). Ciencia, Investigación y Cultura en la biblioteca universitaria actual. Conferencia magistral presentada en la Academia de Ciencias de Cuba. La Habana. 2006. Fecha de consulta: 2012-05-20. Disponible: http://decs.bvs.br/E/homepagee.htm 
Suárez, C., Escalona, E. y Boza, Y. (2012). Algunas consideraciones sobre el proceso de autoevaluación de la actividad científica educacional en las Universidades de Ciencias Pedagógicas, Revista Congreso Universidad, I (2), Fecha de consulta: 2012-06-10. Disponible:

http://200.14.55.210/revista/index.php/congresouniversidad/article/viewFile/85/71

Villamón, M. et al., (2005). Análisis de la visibilidad de las revistas científico-técnicas españolas de ciencias de la actividad física y el deporte. Revista de Psicología del Deporte, 14(2), p. 253-267.

Velásquez, L. (2007). Las redes de investigación virtuales: propuesta de fomento y desarrollo de la cultura investigativa en las instituciones de educación superior. Revista de Universidad y Sociedad del Conocimiento (RUSC), 4(2). Fecha de consulta: $\quad$ 2013-11-1. http://www.uoc.edu/rusc/4/2/dt/esp/velasquez.pdf 